\title{
Kansei Analysis for Robotic Motions in Ubiquitous Environments
}

\author{
Janaka Chaminda Balasuriya ${ }^{1}$, Chandrajith Ashuboda Marasinghe ${ }^{2}$, \\ Keigo Watanabe ${ }^{1}$, and Minetada Osano ${ }^{3}$ \\ ${ }^{1}$ Department of Advanced Systems Control Engineering \\ Saga University, 1-Honjomachi, Saga 840-8502, Japan \\ jcbala@lycos.com, watanabe@me.saga-u.ac.jp \\ 2 Department of Management and Information Systems Science \\ Nagaoka University of Technology, 1603-1 Kamitomioka, Nagaoka, Niigata \\ 940-2188, Japan \\ ashu@kjs.nagaokaut.ac.jp \\ ${ }^{3}$ Software Engineering Lab, Department of Computer Software \\ University of Aizu, Aizu-Wakamatsu, Fukushima, Japan \\ osano@u-aizu.ac.jp
}

\begin{abstract}
Human beings are fascinating creatures. Their behavior and appearance cannot be compared with any other living organism in the world. They have two distinct features with compared to any other living being; unique physical nature and emotions / feelings. Anybody who studies on humans or trying to construct human like machines should consider these two vital facts. When robots are interacting with humans and other objects, they certainly have a safe distance between them and the object. But how can this distance be optimized when interacting with humans; will there be any advantages over achieving this; will it help to improve the condition of robots; can it be a mere constant distance; how will the humans react, are some questions arosed. In order to "humanize" robots, they (robots) should also have certain understating of such emotions that we, humans have. In this research project, authors are trying to "teach" one such human understanding, commonly known as "personal space" to autonomous mobile robots.
\end{abstract}

\section{Introduction}

As Simmons et al. 1] describe, recent research in mobile robot navigation has utilized autonomous mobile robots in service fields. To operate them in an environment with people, it requires more than just localization and navigation. The robots should recognize and act according to human social behavior to share the resources without conflict 2. Sometimes, even when humans interact with each other, it leads to resource conflict. At such times humans use social rules to maintain order $\underline{3}$.

The comfort level of the humans for which the robot is working will be very important if the robot is to do its job effectively. Extensive research is being performed in the area of robotics to improve the conditions of the robots, advancing 
the abilities to do specific tasks, motion planning, etc. But very little work has been performed in trying to understand how people would interact with a robot, how to make them comfortable, factors that make uncomfortable or threatening, methods or ways for robots to indicate their feelings, etc. to analyze the aesthetic qualities of the robots behavior patterns [2].

\subsection{Distance from the Robot to the Vicinity}

As in the very beginning of the mobile robotic systems, there had been some kind of distance or space between the robots to any other object in the vicinity. This was just a mere distance for safety for easy maneuvering and for collapse avoidance. As Stentz [4] and many others had mentioned, this was just a constant of space. This mechanism was quite acceptable for the systems such as transporting, surveillance and monitoring, etc. In other words, such kind of safe distance was good for non-human interacting purposes. Can the same be applied for human interaction? Although it will give some results, it will not enhance or optimize the real requirement in need, i.e. to build up harmonious relationship with humans.

\subsection{Nakauchi Model}

When Nakauchi and Simmons 2] studied about personal space and applied it to moving robots, it was some improvement over the "blind" safe distant. In their mechanism, Nakauchi and Simmons had experimented using human subjects for "correct distance" or "personal space" in order to have pleasant feeling towards the two interacting parties.

This experimentally derived average personal space had an approximate shape of an oval that is larger towards the front. Though those results were approximate, it had been aligned with the values that were reported in the cognitive science literature [5].

\subsection{Walters Experiments}

Another set of experiments were conducted by Walters et al. 6] using adults and children with a robot of mechanistic appearance called PeopleBot ${ }^{R}$ to find the personal space zones, initial distances between robot and humans, etc. the context of the encounters and the human's perception of the robot as a social being. They had found out that the children showed a dominant response to prefer the "social zone" comparable to distances people adopt when talking to other humans. From the adult studies, they found that, a small number of people preferred the "personal zone" though significant minorities deviate from this pattern.

\section{Variation of Personal Space}

Although it is possible to find a personal space for a specific instance of environment, it is highly volatile depending on the two interaction parties and 
not definitely a constant. As Walters et al. 6] suggested, different robot social models, perhaps with very different initial personalities, may be more acceptable to different users (e.g. a discreet servant or even a silent servant, with no obvious initiative or autonomy). They further stated that it probably cannot be assumed that people automatically treat robots socially, apart from simple elements of anthropomorphism as described by Reeves and Nass [7]. A userfriendly robot should automatically refine and adapt its social model (personality) over a longer period of time, depending on information about and feedback from users and the robots own autonomous learning system. For example, adjustments of social distances according to a user's personality trait will be a promising direction (as proposed in [8]) is a promising direction towards a true robot companion that needs to be individualized, personalized and adapt itself to the user 9 .

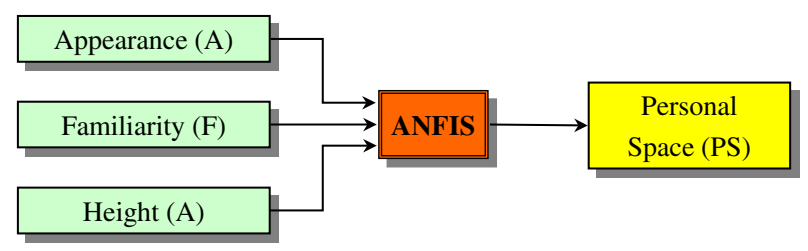

Fig. 1. APS ANFIS block diagram

According to Sack 3] and Malmberg [5], it is reported that the actual size of the personal space at any given instance varies depending on cultural norms and on the task being performed. For a simplified scenario for experimentally analysis; appearance (mainly of the robot), previous acquaintance or familiarity of the either parties, gender, age, height of the bodies, (specially interaction in the standing position), emission of any sound, emotions on the face, carrying objects, etc. were considered to be important. From the list of above, first three: height, appearance and familiarity were considered (as the initial stage for simplicity) to generate an active personal space (APS) determination system and the block diagram is shown in Figure 1.

\section{ANFIS for Personal Space Determination}

Adaptive Neural Fuzzy Inference System or simply ANFIS can be used as a basis for constructing a set of fuzzy if-then rules with appropriate membership functions to generate the desired input-output combination [10]. It is especially useful when needed to apply a fuzzy inference to already collected input-output data pairs for model building, model following, etc. where there are no predetermined model structures based on characteristics of variables in the system. 


\subsection{Gathering Data}

Considering the procedure as Nakauchi and Simmons [2] or the Walters et al. 6] to obtain a sense of personal space for robot / human interaction, a similar experimental condition was constructed. Here a robot (or a model) is kept at the end of a scaled line in a room and a human is asked to move closer to it.

\subsection{Experimental Procedure}

As the experiment proceeds, one human subject is instructed to move towards the robots as if he needs to talk with it. The human subject is asked to be along the scaled line and look at the robot face and move closer to it until he feels safe enough to make conversation with it. In the mean time the robotic model was positioned so as to make its face towards the human subject. During the whole time of the experiment, the robot did not do anything and the human subject did all the active tasks of walking, thinking, etc. The robot and the human subject, one person at a time, were supposed to interact at one specific duration of time and it ended once the human subject stops in front of the robot. Then the distance between the two parties was obtained by using a camera or by direct human observer (who reached the two parties once they got stabilized). The human subject had no previous experience with the robot and the authors wanted the human subjects to be curious as well as cautioned about the robot that they are going to meet. In other wards human subjects had no idea what kind of robotic system that they are going to face with or any capabilities that it possesses until they meet the robot.

The robots and the robotic models used in these experiments are 'KooruKun' (PA10 robotic manipulator), 'BoxChyan' (robotic model), and 'BasinKun' (previously known as 'CHRCarry Hospital Robot' re-used with several modifications). The first one was a stationary robot with $200 \mathrm{~cm}$ in height and $20 \mathrm{~cm}$ average diameter, next was a movable robot model with $100 \mathrm{~cm}$ height, $50 \mathrm{~cm}$ diameter and around $3 \mathrm{Kg}$, and the last is also a movable robot with $170 \mathrm{~cm}$ height, generalized circular diameter of $60 \mathrm{~cm}$ and weight of about $25 \mathrm{Kg}$. The data gathered are grouped for training, checking and testing for the ANFIS and are described in later.

\section{Inputs and Parameters}

Out of the three variable parameters considered, height (of the human) and familiarity were two straight-forward measurements while appearance was taken as a collective decision.

\subsection{Input Variable 'height $(\mathrm{H})$ '}

The height of the human subject is considered in this input variable. The universe of disclose of the input variable 'height $(\mathrm{H})^{\prime}$ ' was considered to be $50 \mathrm{~cm}$ to $200 \mathrm{~cm}$, having three membership functions 'big (B)', 'medium (M)', and 'small (S)'. Bell Shape was considered. 


\subsection{Input Variable 'familiarity (F)'}

Previous interactions with the same robot / human pair are considered in this input variable. Keeping in mind that more interactions means more familiar with each other, this variable is set. For the first time of interaction having the value of 1 and 5 times or more having the value of 5 for the input assessment. The universe of disclose of the input variable 'familiarity ( $\mathrm{F}$ )' was considered as 1 to 5 (without any units), having three membership functions 'big (B)', 'medium $(\mathrm{M})$ ', and 'small (S)'. Bell Shape was considered.

\subsection{Input Variable 'appearance (A)'}

The robots' outer appearance to that of a human is considered in this input variable. Humans are more like to reach one of their own looking rather than to that of very peculiar shaped objects. Human like robot get the more closer feeling with respect to the other crude or rather machine looking robot. The universe of disclose of the input variable 'appearance (A)' was considered to be 1 to 5 (without any units), having three membership functions 'big (B)', 'medium $(\mathrm{M})$ ', and 'small (S)'. Bell Shape was considered. Here the appearance value for the 'KooruKun' robot was given as 1, for the 'BoxChyan' as 2, and 'BasinKun' as 5 . Although more human like robots were required to get the records, at the time of the experiment, such robots were not available in the laboratory. (Hence the universe of disclose was 1 to 5$)$.

\subsection{Output Variable 'personal space (PS)'}

Considering the above three inputs that will generate 27 rules in total and each having unity weight for each rule, the output of the ANFIS is obtained using the weighted average defuzzification.

\section{ANFIS Architecture}

The architecture of the APS determination network is illustrated in Figure 2, Layer (I) to Layer (III) represent the antecedent part of the fuzzy neural network, whereas Layer (V) and Layer (VI) represent the consequence part [1].

As shown in Figure 2] the domain of discourse of height $(\mathrm{H})$ is described by fuzzy variable $H$ with $p$ number of linguistic values $(p=3)$, the domain of discourse of appearance (A) is described by fuzzy variable $A$ with $q$ number of linguistic values $(q=3)$, and the domain of discourse of familiarity $(\mathrm{F})$ is described by fuzzy variable $F$ with $r$ number of linguistic values $(r=3)$. Hence each input variable is unique in the sense of domain of discourse. It is assumed that each node of the same layer has a similar function, as described below. Here we denote the output of the $i$ th node in layer $(\mathrm{X})$ as $O_{(X), i}$.

Layer (I)

Layer (I) consists of three types of nodes; height (H), appearance (A) and familiarity $(\mathrm{F})$. The current value of height $(\mathrm{H})$, i.e. the crisp input to the height 


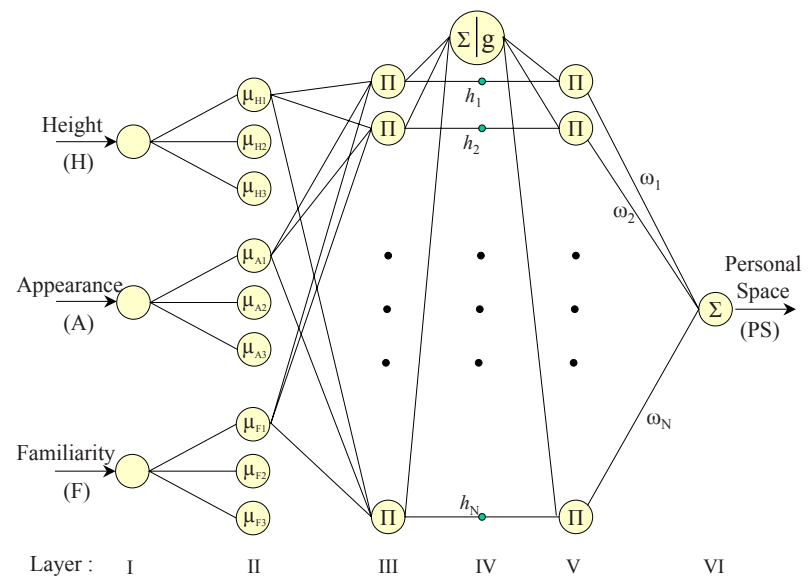

Fig. 2. APS ANFIS architecture

node is $H_{i}$, appearance node is $A_{j}$ and familiarity node is $F_{k}$. No computation is carried out at this layer.

Layer (II)

This layer acts as the fuzzification layer of the fuzzy neural network. At this layer, the output of a node connected to the current value of variable input acquires the fuzzy membership value of the universe of discourse. Every node $i$, where $i=1, \cdots, p$ (or $q$ or $r$ ), in this layer is an adaptive node with a node function

$$
O_{I I, i}=\mu_{X_{i}}(x)
$$

where $x$ is the input to node $i$, and $X_{i}$ is the linguistic label (big, medium, small, etc.) associated with this node function. In other words, $O_{I I, i}$ is the membership function of $X_{i}$ and it specifies the degree to which the given $x$ satisfied the quantifier $X_{i}$. Hence the output from the 2nd layer will be:

$$
\begin{aligned}
& O_{I I, p}=\mu_{H_{i}}\left(H_{i}\right) \\
& O_{I I, q}=\mu_{A_{q}}\left(A_{j}\right) \\
& O_{I I, r}=\mu_{F_{r}}\left(F_{k}\right)
\end{aligned}
$$

for height, appearance and familiarity respectively.

Layer (III)

In this layer, the nodes labeled as $\Pi$ compute the T-norm of the antecedent part. Thus the rule evaluates the conditions of the inputs and they are continued to the layer $(\mathrm{V})$ for normalization. The output of any node $t$, where $t=1, \cdots, N$, where $N=p * q * r$, in this layer is described by the following equation:

$$
O_{I I I, t}=h_{t}=\mu_{H_{i}}\left(H_{i}\right) * \mu_{A_{q}}\left(A_{j}\right) * \mu_{F_{r}}\left(F_{k}\right)
$$


where $h_{t}$ represents the firing strength of the $t$ th rule and there are $N$ such rules as total.

Layer (IV)

The first node of layer (IV) at fuzzy neural network, which has symbols $\sum$ and $g$, generates the output through the following function:

$$
g(x)=\frac{1}{x}
$$

with a linear summed input. Then the output of the first node of layer IV is given by

$$
O_{I V, 1}=\frac{1}{\sum_{t=1}^{N} h_{t}}
$$

Other nodes just carry forward the outputs of previous nodes to the next layer.

Layer ( $V)$

This layer normalizes the fired rule values. Each node labeled as $\Pi$ in this layer multiplies the value carried forward by previous node with the output of the first node at Layer (IV). Then the output of any $m$ th node of this layer can be given by the following equation:

$$
O_{V, m}=\frac{h_{m}}{\sum_{t=1}^{N} h_{t}}
$$

Layer (VI)

Layer (VI) is the defuzzification layer of the fuzzy neural network. The node labeled as $\sum$ in this layer calculates the overall output as the summation of all incoming signals. Then the personal distance value for certain input variables is given by:

$$
O_{V I}=\text { Personal_Diatance }=\frac{\sum_{m=1}^{N} w_{m} h_{m}}{\sum_{n=1}^{N} h_{n}}
$$

where $w_{m}$ denotes a constant value in the consequence part of the $m$ th rule. The overall output is the weighted mean of $w_{m}$ with respect to the weight $h_{m}$.

The connection weights are trained by applying the hybrid algorithm. The error tolerance was set to zero.

The error is calculated by comparing the output of the expert knowledge with that of fuzzy neural network for the same input data, $x$. The adaptation of the $m$ th weight, $w_{m}$, at the $l$ th time step is given by the following equation: 


$$
w_{m}(l+1)=w_{m}(l)+\gamma\left[y_{d}-y_{a}\right] \frac{h_{m}}{\sum_{n=1}^{N} h_{n}}
$$

where $\gamma$ represents a small positive learning rate, and $y_{d}$ and $y_{a}$ represent the desired output and actual output respectively for the personal space value selected for the training.

\section{Training, Checking and Testing Data Sets}

From the collected data from the experiments, data were rearranged in to 3 groups for training, checking and testing purposes of the APS ANFIS.

\subsection{Train Data Set}

Train data set was obtained by grouping the input variable 'height $(\mathrm{H})$ '. First, the height was categorized in to 5 groups as:

- $161 \mathrm{~cm}$ to $165 \mathrm{~cm}$

$-166 \mathrm{~cm}$ to $170 \mathrm{~cm}$

- $171 \mathrm{~cm}$ to $175 \mathrm{~cm}$

- $176 \mathrm{~cm}$ to $180 \mathrm{~cm}$

$-181 \mathrm{~cm}$ to $185 \mathrm{~cm}$

Then under each column, average is taken. The results thus obtained were used as the train data set.

\subsection{Check Data Set}

In order to optimize the ANFIS and to make a slighter change in the trained data set, following equation was considered when obtaining the check data set. I.e. considering a particular column, average value is subtracted from the maximum value from the gathered data. Then half of that value is added to the minimum value of the gathered data in the same column.

$$
\text { ChkDataValue }=\left[\frac{\text { Max.Data }- \text { Ave.Data }}{2}\right]+\text { Min.Data }
$$

\subsection{Test Data Set}

Considering the original data from the experiments, very close to the training and checking data sets, one data entry is selected for each category. By this selection, another set of data is obtained for testing purpose of the ANFIS.

The trained ANFIS output with train data and check data are shown in Figures 3 and 4. 


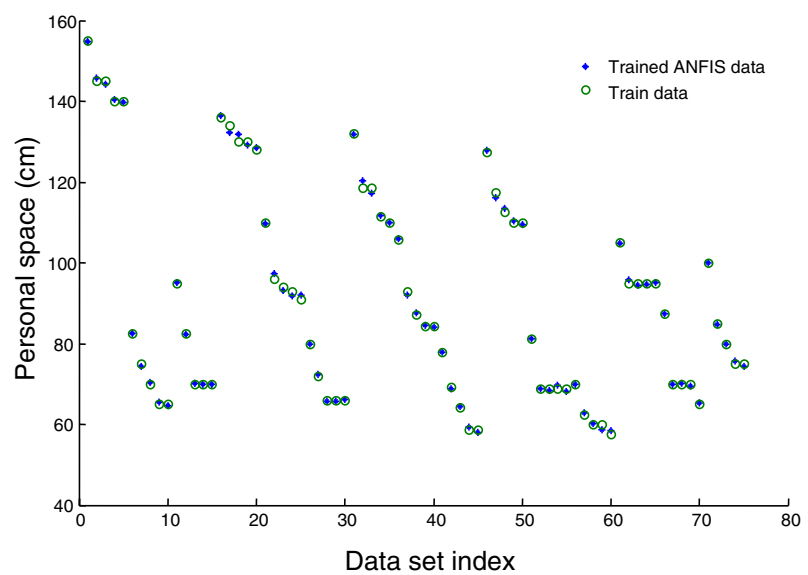

Fig. 3. Trained FIS with train data

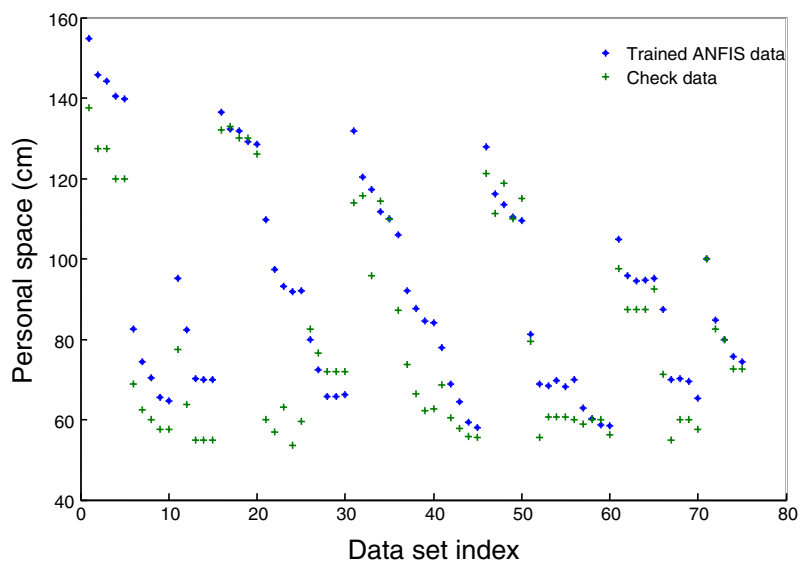

Fig. 4. Trained FIS with check data

\section{Summary}

In this research project, a determination system of an active personal space has been analyzed. This can be considered as the first step for constructing an active personal space system for any robot in any environment. But for a target as such, many experiments in vast environmental situations should have to be involved. It is a must to obtain similar data with the so-called humanoids to make this experiment complete. Further, more sophisticated supportive equipment such as high speed processing units for human recognition, memory acquisition and manipulation, image processing, etc. should be coupled. This system gave encouraging results in an offline mode with limited facilities. Authors are planning 
to make the current system more realistic and get the functioning in a real time mode, and are continuously working on it.

\section{References}

1. Simmons, R., Goodwin, R., Haigh, K.Z., Koenig, S., O'Sullivan, J.: A layered architechture for office dilivery robots. In: Proc. of Autonomous Agents, pp. 245252 (1997)

2. Nakauchi, Y., Simmons, R.: A social behavioral robot that stands in line. Autonomous Robots 12, 313-324m (2002)

3. Sack, R.: Human Territory. Cambridge University Press, Cambridge (1986)

4. Stentz, A.: Map-based strategies for robot navigation in unknown environments. In: Proc. of AAAI, pp. 110-116 (1996)

5. Malmberg, M.: Human Territoriality: Survey of behavioural territories in man with preliminary analysis and discussion of meaning. Mouton Publishers (1980)

6. Walters, M.L., Dautenhahn, K., Koay, K.L., Kaouri, C., Boekhorst, R., Nehaniv, C., Werry, I., Lee, D.: Close encounters: spatial distances between people and a robot of mechanistic appearance. In: Proc. of 5th IEEE - RAS Int. Conf. on Humanoid Robots, December 2005, pp. 450-455 (2005)

7. Reeves, B., Nass, C.: The Media Equation: How people treat computers, television and new media like real people and places. Cambridge University Press, Cambridge (1998) ISBN 157586052x

8. Walters, M L, Dautenhahn, K., te Boekhorst, R., Koay, K L, Kaouri, C., Woods, S., Nehaniv, C L, Lee, D., Werry, I.: The influence of subjects' personality traits on personal spatial zones in a human-robot interaction experiment. In: Proc. of 14th Annual Workshop on Robot and Human Interactive Communication (IEEE Ro-man 2005), Tennessee, USA, August 2005, pp. 347-352 (2005)

9. Dautenhahn, K.: Robots we like to live with - A developmental perspective on a personalized life-long robot companion. In: Proc. of 13th Annual Workshop on Robot and Human Interactive Communication (IEEE Ro-Man 2004), Okayama, Japan, September 2004, pp. 17-22 (2004)

10. Jang, J.S.R.: ANFIS: Adaptive-network-based fuzzy inference system. In: Proc. of IEEE SMC, May/June 1993, vol. 23(3), pp. 665-685 (1993)

11. Jang, J.S.R., Sun, C.T.: Neuro-fuzzy modeling and control. In: Proc. of the IEEE, March 1995, vol. 83(3), pp. 378-406 (1995) 